University of Wollongong

Research Online

Faculty of Engineering and Information

Faculty of Engineering and Information

Sciences - Papers: Part A

Sciences

$1-1-2013$

\title{
A relaxation strategy with fuzzy constraints for supplier selection in a power market
}

Dien Tuan Le

University of Wollongong, dt1844@uowmail.edu.au

Minjie Zhang

University of Wollongong, minjie@uow.edu.au

Fenghui Ren

University of Wollongong, fren@uow.edu.au

Follow this and additional works at: https://ro.uow.edu.au/eispapers

Part of the Engineering Commons, and the Science and Technology Studies Commons

Research Online is the open access institutional repository for the University of Wollongong. For further information contact the UOW Library: research-pubs@uow.edu.au 


\title{
A relaxation strategy with fuzzy constraints for supplier selection in a power market
}

\begin{abstract}
A power market is a special kind of e-markets. In a power market, all trading processes are related to three parties: buyers, suppli- ers and brokers. A broker acts as middlemen between buyers and sup- pliers in a trading process. In a power market, how to select a potential supplier for a buyer through a broker based on the buyer's requirements is a challenging research problem. This paper proposes relaxation strat- egy with fuzzy constraints for supplier selection. The strategy includes three components, i.e., a supplier selection, a fuzzy constraint relaxation, and a decision making. The major contributions of this paper are that (1) the trading process between buyers and suppliers through brokers is modeled by using fuzzy constraints through the consideration of multiple attributes of the buyer's requirements as well as potential power suppli- ers; and (2) a buyer can utilize a relaxation with fuzzy constraints to change its requirements in dicult situations when a broker cannot nd any supplier to satisfy a buyer's requirements. Experimental results show that our approach is successfully applied in a simulated power market.
\end{abstract}

\section{Keywords}

power, selection, supplier, strategy, fuzzy, market, constraints, relaxation

\section{Disciplines}

Engineering | Science and Technology Studies

\section{Publication Details}

Le, D., Zhang, M. \& Ren, F. (2013). A relaxation strategy with fuzzy constraints for supplier selection in a power market. In T. Ito, M. Zhang \& X. Tang (Eds.), The International Workshop on Smart Simulation and Modelling for Complex Systems (SSMCS 2013) (pp. 62-73). China: IJCAI. 


\title{
A Relaxation Strategy with Fuzzy Constraints for Supplier Selection in a Power Market
}

\author{
Dien Tuan Le, Minjie Zhang and Fenghui Ren \\ School of Computer Science and Software Engineering \\ University of Wollongong,Wollongong, 2500, NSW, Australia \\ dt1844@uowmail.edu .au, \{minjie, fren\}@uow.edu.au
}

\begin{abstract}
A power market is a special kind of e-markets. In a power market, all trading processes are related to three parties: buyers, suppliers and brokers. A broker acts as middlemen between buyers and suppliers in a trading process. In a power market, how to select a potential supplier for a buyer through a broker based on the buyer's requirements is a challenging research problem. This paper proposes relaxation strategy with fuzzy constraints for supplier selection. The strategy includes three components, i.e., a supplier selection, a fuzzy constraint relaxation, and a decision making. The major contributions of this paper are that (1) the trading process between buyers and suppliers through brokers is modeled by using fuzzy constraints through the consideration of multiple attributes of the buyer's requirements as well as potential power suppliers; and (2) a buyer can utilize a relaxation with fuzzy constraints to change its requirements in difficult situations when a broker cannot find any supplier to satisfy a buyer's requirements. Experimental results show that our approach is successfully applied in a simulated power market.
\end{abstract}

Keywords: Supplier selection, relaxation strategy, fuzzy constraints, power market.

\section{Introduction}

E-markets are virtual marketplaces where buyers and suppliers meet to exchange information about price, products and service offerings, and to negotiate and carry out business transactions [1]. A power market is a kind of e-markets with specifications. In a power market, a buyer cannot reach a contract with a supplier directly and any supplier selection must go through a broker. Thus, in a power market involving three parties, it is hard to apply game theory-based negotiation approaches, which have been successfully applied in many e-markets, to reach an agreement in trading processes. In addition, because of electricity energy's constraints from policies of companies or organizations, it is difficult for buyers and suppliers to use automated negotiation approaches [3],[8],[9] or auction mechanisms [7],[12]. Due to the involvement of third party (brokers) [5], a broker in a power market acts as middlemen between buyers and suppliers to help a buyer identify a potential supplier in order to reach an electricity supply contract. 
The study of brokers, acting as the third party of a trading process in emarkets, has been examined by many researchers in recent years. Sarma et al. [11] provided an efficient algorithm to compute the equilibrium for a related game of price in a trading process between buyers and sellers through brokers. Blume et al. [2] studied the trading process in general e-markets between buyers and sellers through a layer of intermediaries. Rubenstein et al. [10] proposed a market model with three types of agents, i.e., sellers, buyers, and middlemen, and analyzed steady state conditions in such markets. Gale et al. [4] analyzed a network model of exchange, in which trading is intermediated. Due to the special features of power markets, these approaches are facing two main challenges in power markets: (1) every trading process between buyers and suppliers in a power market is related to a broker, so a broker plays an important role in the supplier selection; and (2) due to policies of organizations or companies, suppliers cannot make any concession to a buyer.

To address the above challenges, we develop a relaxation strategy with fuzzy constraints to select a potential power supplier to agree on an electricity supply contract through a broker. In particular, our approach uses prioritized fuzzy constraints to present trade-offs between the different possible values of attributes and to indicate how relaxations should be made when they are necessary. The major contributions of this paper are that (1) the trading process between buyers and suppliers through brokers is successfully addressed based on fuzzy constraints for multiple attributes of buyer's requirements as well as potential power suppliers; and (2) a buyer utilizes a relaxation strategy with fuzzy constraints to change its requirements when a broker cannot find any supplier to meet a buyer's requirements. Experimental results demonstrate the good performance of the proposed approach in terms of satisfaction of buyer's requirements.

The rest of this paper is organized as follows. The problem description and definitions are presented in Section 2. A relaxation strategy with fuzzy constraints to select a potential power supplier is introduced in Section 3. An experiment is presented in Section 4. Section 5 concludes this paper and points to our future work.

\section{Problem Description and Definitions}

In general, there are three parties involving in a power market, i.e., suppliers, buyers and brokers. One objective of the trading process between a buyer and suppliers through a broker is to select a potential supplier for the buyer. To achieve the objective, the three parties in the trading process have to follow certain rules. (1) multiple suppliers provide their electricity to the power market; (2) due to policies of companies or organizations, suppliers cannot make any concession to buyers; and (3) a buyer's requirements must be satisfied by suppliers. Before elaborating the details of the approach, it is necessary to define the scope of this research and provide some necessary definitions.

A buyer agent is considered as an electricity consumer who would like to find a potential power supplier and agree on a contract. 
Definition 1. A buyer agent $B_{i}$ is defined as a 4-tuple $B_{i}=\langle I D, E E R, \alpha, \lambda\rangle$, where $I D$ is the buyer agent's identification, EER indicates the electricity energy request (see Definition 2), $\alpha$ is the acceptability threshold of $B_{i}$, and $\lambda$ is the concession threshold of $B_{i}$.

Definition 2. An electricity energy request is represented by EER and is defined by the following format.

$$
E E R=\left(\begin{array}{cccc}
A_{1} & A_{2} & \ldots & A_{n} \\
C_{1} & C_{2} & \ldots & C_{n} \\
W_{1} & W_{2} & \ldots & W_{n}
\end{array}\right),
$$

where $A_{i}$ indicates the $i^{\text {th }}$ attribute name, $C_{i}$ is the constraint value of $A_{i}$ and $W_{i}$ is the priority value of $A_{i}, 1 \leq W_{i} \leq n . W_{i}=1$ indicates the lowest priority and $W_{i}=n$ indicates the highest priority.

A supplier agent is considered as a company or an organization and its responsibility is to sell electricity to buyer agents in a power market.

Definition 3. A supplier agent $S_{i}$ is defined as a 3-tuple $S_{i}=\langle I D, E R, B O\rangle$, where $I D$ is the identification of $S_{i}, E R$ indicates an electricity resource (see Definition 4) provided by $S_{i}$, and $B O$ is a bonus value which a supplier agent may use to attract a buyer agent to purchase electricity.

The electricity resource is provided by a supplier agent. Due to company or organization policies, an offer from a supplier agent contains some constraints to electricity resource such as constraints of price and time.

Definition 4. An electricity resource provided by a supplier agent $S_{i}$ is presented by ER and is defined by the following format.

$$
E R=\left(\begin{array}{cccc}
A_{1} & A_{2} & \ldots & A_{n} \\
C_{1} & C_{2} & \ldots & C_{n}
\end{array}\right),
$$

where $A_{i}$ is the $i^{\text {th }}$ attribute name and $C_{i}$ is the constraint value of $A_{i}$ provided by $S_{i}$.

A broker agent acts as the third party in a trading process between a buyer agent and supplier agents. A broker agent's responsibility is to select the most suitable supplier agent to meet a buyer agent's requirements.

Definition 5. A broker agent $B R_{i}$ is defined as $B R_{i}=<\mathbf{S}, B_{i}, r>$, where $\mathbf{S}$ is a set of supplier agents, which sell electricity to a buyer agent $B_{i}, r$ is a reward that $B R_{i}$ can get from a supplier agent.

\section{A Relaxation Strategy with Fuzzy Constraints for Supplier Selection}

Our relaxation strategy includes the three main components: (1) the supplier selection; (2) the relaxation with fuzzy constraints; and (3) the decision making. In this section, the principle of the whole trading process is introduced in subsection 3.1. Then the three main components are presented in details in three subsections, respectively. 


\subsection{The principle of the whole trading process}

Background A trading process between a buyer agent and supplier agents is conducted through a broker agent to achieve an agreement by using certain strategies. In our strategy, a buyer agent utilizes the relaxation with fuzzy constraints to change its requirement in difficult situations. The broker agent relies on a reward from supplier agents to select the most suitable supplier agent for the buyer agent. Supplier agents use a bonus policy to attract the buyer agent to purchase their electricity. The principle of the whole trading process between a buyer agent and supplier agents through a broker agent in our approach is presented in Fig. 1.

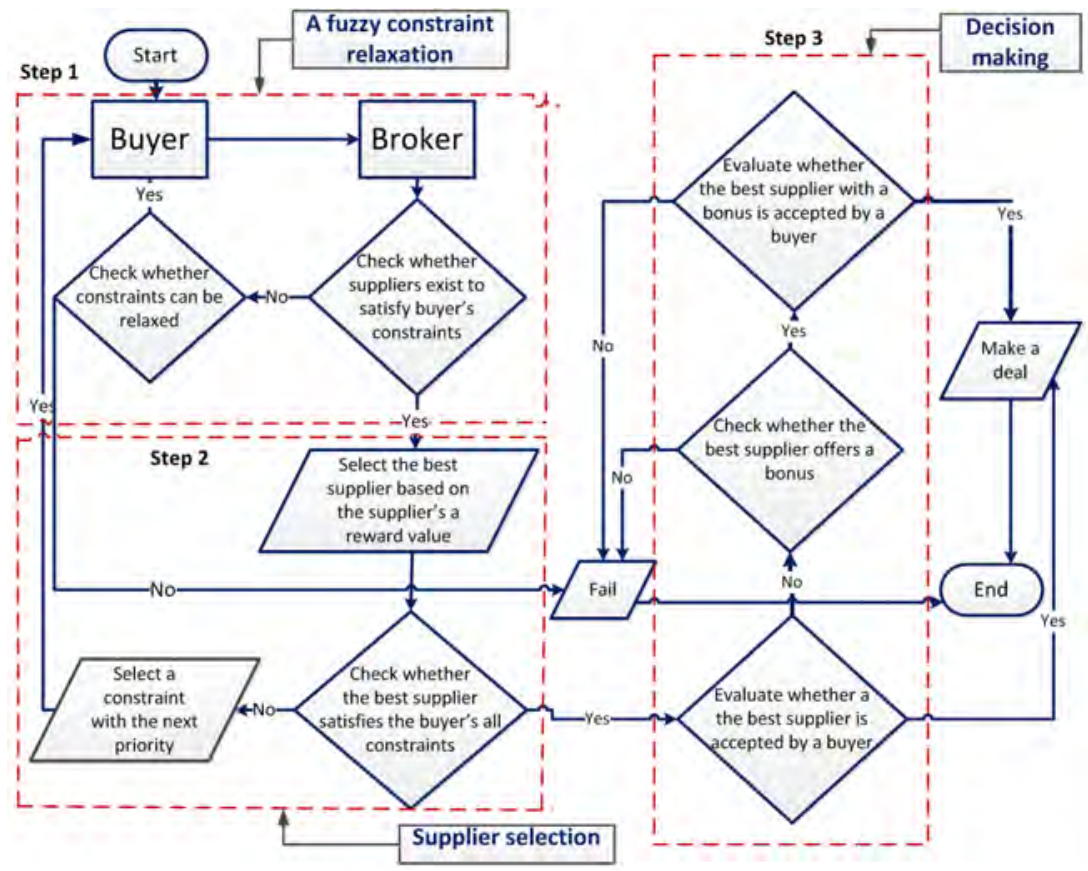

Fig. 1. Diagram of the principle

Step 1: The buyer agent selects a constraint of attributes with the highest priority from its requirements and sends the constraint to the broker agent. Based on the buyer agent's constraint, the broker agent searches for supplier agents. If the broker agent cannot find any supplier agent, the broker agent checks whether the buyer agent's constraints can be relaxed. If the relaxation is not applied, the trading process is terminated. Otherwise, the buyer agent selects a relaxed constraint and sends it to the broker agent again. This procedure will be repeated until the broker agent finds supplier agents to satisfy the buyer agent's constraints or the trading is terminated.

Step 2: Once the broker agent finds suitable supplier agents, it will select the most suitable supplier agent based on the suitable supplier agents' rewards and 
send the selected supplier agent to the buyer agent. The buyer agent checks whether the most suitable supplier agent satisfies the buyer agent's other constraints. If there are more constraints, the buyer agent selects the next highest priority constraints and sends it to the broker agent again, and the process goes to step 1. Otherwise, the buyer agent evaluates whether the most suitable supplier agent is acceptable.

Step 3: If the buyer agent accepts the most suitable supplier agent, the trading process makes a deal. Otherwise, the buyer agent requires the broker agent to check whether the most suitable supplier agent offers a bonus. If the most suitable supplier agent does not offer a bonus, the trading process between the buyer agent and the broker agent is terminated. Otherwise, the buyer agent evaluates the most suitable supplier agent with a bonus again to make a decision.

Formal description A formal representation of the process of potential supplier selection is described by Algorithm 1.

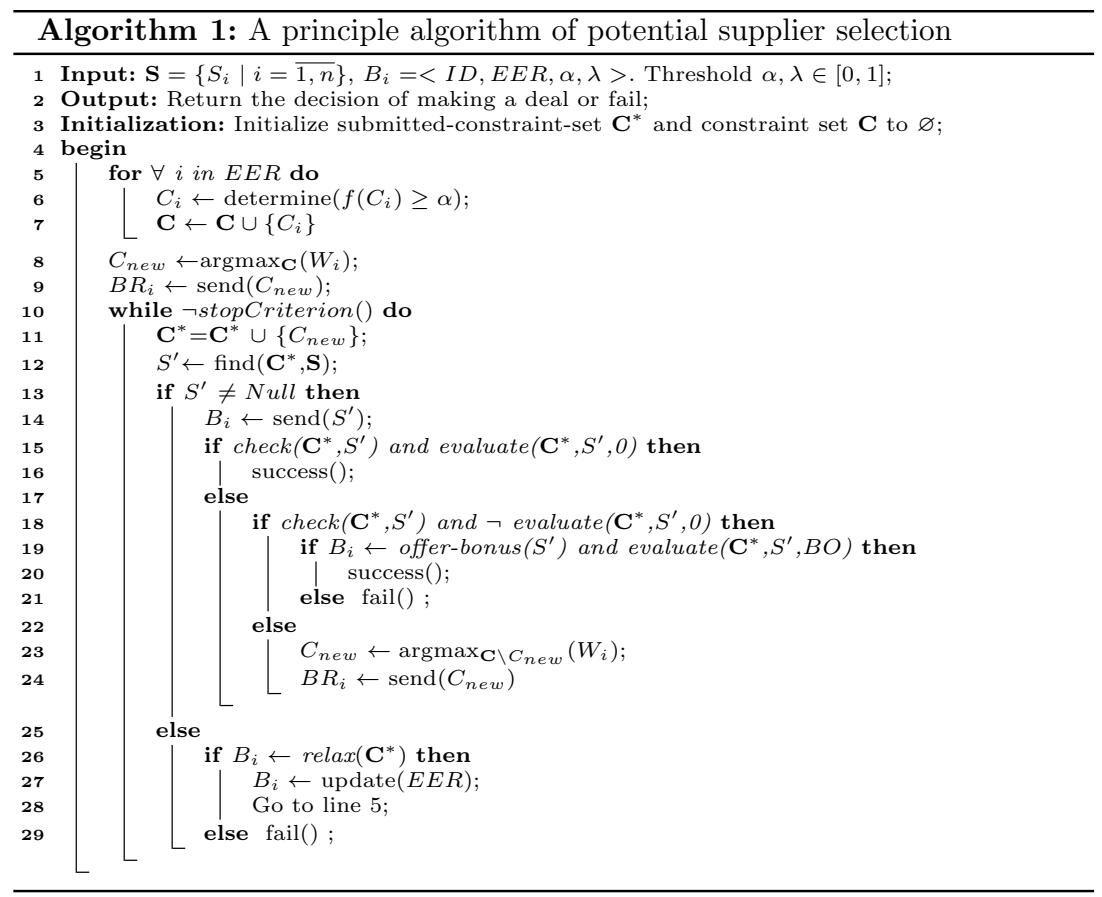

The algorithm shows all cases of the trading process between a buyer agent $B_{i}$ and a set of supplier agents $\mathbf{S}$ through a broker agent $B R_{i}$ based on the $B_{i}$ 's requirements, an acceptability threshold and a concession threshold (line 1). The output of the algorithm can be either 'deal' or 'fail' (line 2).

First, $B_{i}$ uses its acceptability threshold to determine each constraint value of an attribute in $E E R$ (lines 6-7). Then $B_{i}$ selects a constraint of an attribute in $E E R$ with the highest priority and sends it to $B R_{i}$ (lines 8-9). $B R_{i}$ finds the most suitable supplier agent to satisfy $B_{i}$ 's requirements (line 12) by using 
'find' function described in subsection 3.2. The results from $B R_{i}$ are presented as follows.

If $B R_{i}$ finds the most suitable supplier agent, $B R_{i}$ sends the most suitable supplier agent to $B_{i}$ (line 14). Then, $B_{i}$ verifies whether the most suitable supplier agent satisfies $B_{i}$ 's requirements and evaluation (line 15) by using 'evaluation' function described in Subsection 3.4. There are three cases in this situation. (1) if $B_{i}$ 's requirements and evaluation are acceptable, a deal is made (line 16). (2) if $B_{i}$ 's requirements are satisfied but $B_{i}$ 's evaluation is not acceptable, $B_{i}$ verifies whether the most suitable supplier agent offers a bonus. If the most suitable supplier agent offers the bonus and $B_{i}$ 's evaluation with a bonus is acceptable, the trading process between $B_{i}$ and $B R_{i}$ makes a deal. (lines 18-20). Otherwise, the trading process between $B_{i}$ and $B R_{i}$ is terminated (line 21). (3) if $B_{i}$ 's requirements are not satisfied, $B_{i}$ selects a constraint of attributes with the next highest priority in the $E E R$ and sends it to $B R_{i}$ (lines 23-24). Thus, $B R_{i}$ has to find suitable suppliers again with the new constraints.

If $B R_{i}$ does not find any suitable supplier agent, which satisfies $B_{i}$ 's requirements, $B_{i}$ has to relax its requirements (line 26) by using 'relaxation' function described in Subsection 3.3. In particular, if a constraint of an attribute is relaxed by $B_{i}, B_{i}$ has to update its $E E R$ and the algorithm runs again with the updated $E E R$ (lines 27-28). Otherwise, the trading process is terminated (line 29).

The three major components of the proposed approach are introduced in detail in the following three subsections, respectively.

\subsection{Supplier selection}

When a broker agent receives the buyer agent's requirements, the broker agent starts to find the most suitable supplier agent for a buyer agent. The 'find' function, displayed in line 12 of Algorithm 1, is shown in Algorithm 2 as follows.

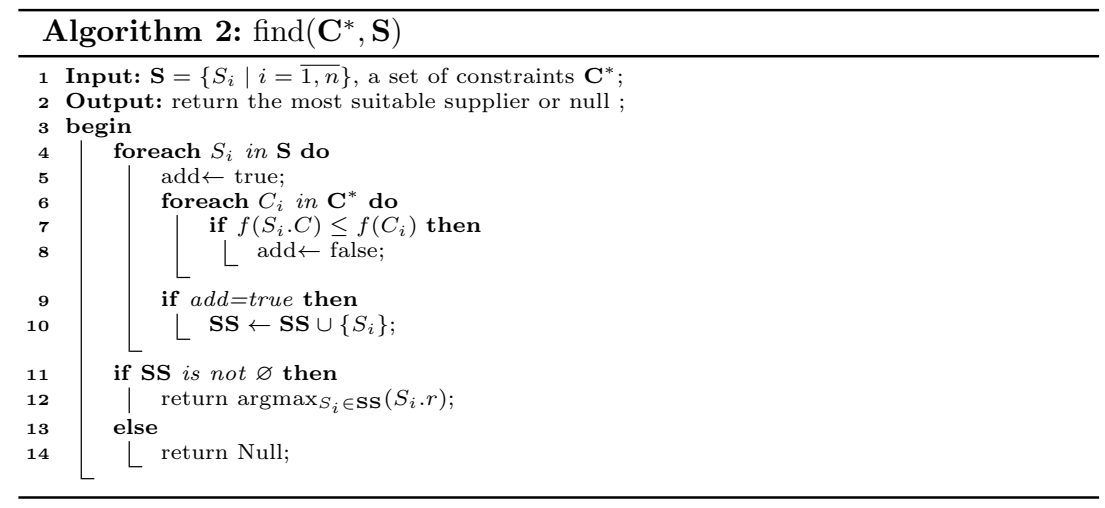

The algorithm 2 shows how to select the most suitable supplier agent based on a set of suppliers $\mathbf{S}$, a set of constraints called $\mathbf{C}^{*}$ which has been submitted 
to $B R_{i}$ during the selection stage (line 1$)$. The output of the algorithm can be either 'the most suitable supplier' or 'null' (line 2). First, $B R_{i}$ selects a suitable supplier set, which satisfies $B_{i}$ 's requirements (lines 4-10). Then, the most suitable supplier agent is selected from the supplier set based on a maximal reward value from supplier agents. If $B R_{i}$ finds the most suitable supplier agent, $B R_{i}$ sends it to $B_{i}$ (line 12). Otherwise, $B R_{i}$ cannot find any supplier agent which satisfies $B_{i}$ 's requirements (line 14).

\subsection{A fuzzy constraint relaxation}

If $B R_{i}$ cannot find any $S_{i}$, which satisfies $B_{i}$ 's requirements, $B R_{i}$ requests $B_{i}$ to consider relaxing its requirements. The 'relaxation' function, displayed in line 26 of Algorithm 1, will be activated. The relaxation function is shown in Algorithm 3.

The algorithm shows how to carry out the relaxation based on a set of constraints called $\mathbf{C}^{*}$, which has been submitted to $B R_{i}$ so far and the concession threshold $\lambda$ (line 1). The output of the algorithm can be either 'a selected constraint for the relaxation' or 'false of the relaxation' (line 2 ). The algorithm proceeds as follows.

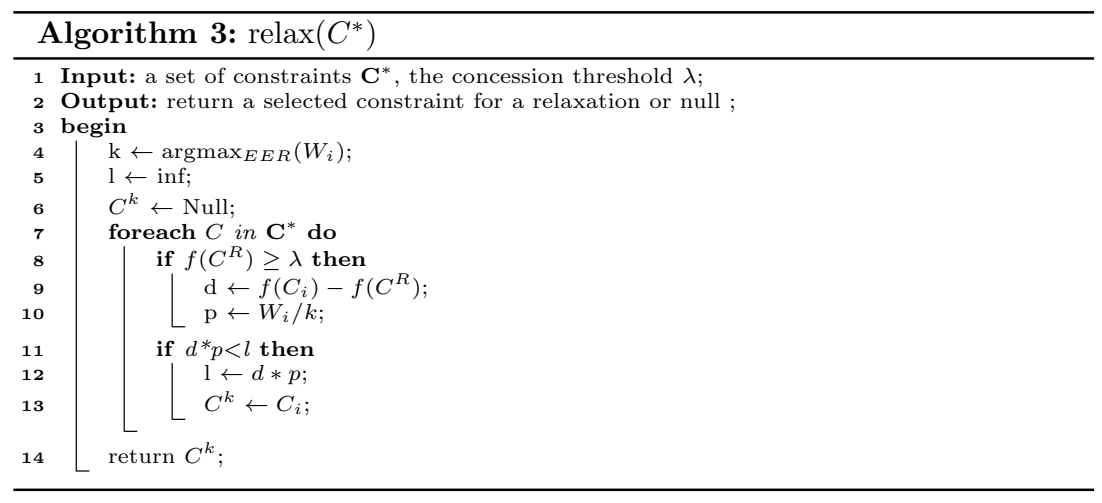

After determining the highest priority in $E E R$ (line 4), $B_{i}$ checks whether each constraint of an attribute in $C^{*}$ is satisfied for the relaxation. This means that $B_{i}$ determines the degrees of satisfaction for the relaxation of each constraint. When constraint $\mathrm{C}$ of an attribute is decreased to the next highest satisfaction degree, the decreased constraint is named $C^{R}$. If a satisfaction degree of a relaxed constraint $f\left(C^{R}\right)$ is less than its concession threshold $\lambda$, the relaxation of the constraint is not permitted. Otherwise, the constraint is considered for a relaxation. The process of the relaxation is illustrated as follows. First, $B_{i}$ calculates a decreased satisfaction degree (line 9) and a relative priority degree (line 10) for each constraint of an attribute in $C^{*}$. Then, a lost benefit value for each constraint after relaxation is calculated from a decreased satisfaction degree and a relative priority degree. Based on a lost benefit value for each relaxed constraint, $B_{i}$ selects a constraint for a relaxation with the smallest lost benefit to $B_{i}$ (lines 12-13). 


\subsection{Decision making}

The 'evaluation' function, displayed in line 15 of Algorithm 1, is shown in Algorithm 4. Algorithm 4 presents how to evaluate the most suitable supplier agent based on $B_{i}$ 's updated $E E R$, the acceptability threshold, the bonus from the most suitable supplier agent (line 1). The output of the algorithm can be either 'acceptability' or 'unacceptability' (line 2). The algorithm proceeds as follows.

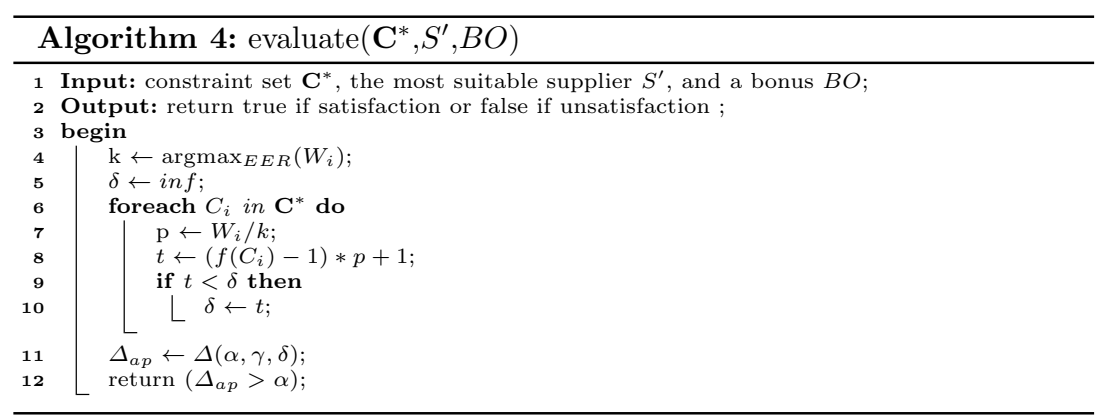

$B_{i}$ calculates an acceptability degree called $\Delta_{a p}$ to compare to $\alpha$. The acceptability degree is related to three parameters $\delta, \gamma$, and $\alpha[6]$. Parameter $\delta \in[0,1]$ is called the overall satisfaction degree and is calculated from $B_{i}$ 's updated $E E R$. To calculate $\delta$ value, we calculate corresponding suitable degree $t_{i}$ for each constraint $C_{i}$ (lines 7-8). Then, $\delta$ value is $\min \left\{t_{i}\right\}$ (line 10). Parameter $\gamma \in[0,1]$ is the satisfactory degree of a bonus from $S^{\prime}$. Parameter $\alpha$ is the acceptability threshold of $B_{i}$. Based on $\delta, \gamma$, and $\alpha[6], \Delta_{a p}$ is calculated from Equation 3 as follows (line 11).

$$
\Delta(\alpha, \gamma, \delta)=\frac{(1-\alpha) \delta((1-\alpha) \gamma+\alpha)}{(1-\alpha) \delta((1-\alpha) \gamma+\alpha)+\alpha(1-\delta)(1-((1-\alpha) \gamma+\alpha))}
$$

If $\Delta_{a p}$ is more than $\alpha$, the most suitable supplier agent is acceptable. Otherwise, the most suitable supplier agent is unacceptable (line 12).

\section{Experiment}

In this section, we illustrate our experimental results on relaxation strategy with fuzzy constraints for supplier selection in the power market. Subsection 4.1 introduces the experimental setting. Subsection 4.2 demonstrates the experimental results.

\subsection{Experiment setting}

The experiment settings include the settings for the buyer agent, supplier agents and the broker agent. 
Supplier setting: The simulation contains six supplier agents and considers four attributes: price, electricity usage on weekdays, electricity usage on weekends and early withdrawal penalty. The detail contents of each supplier are presented in Table 1. Supplier agents use a bonus to attract buyer agents to purchase their electricity. In particular, five of the six supplier agents offer a bonus for the buyer agent and the satisfaction degrees of a bonus for 'gift' and 'free sign up fee' are set as $80 \%$ and $10 \%$, respectively.

\begin{tabular}{|c|c|c|c|c|c||}
\hline Supplier & $\begin{array}{c}\text { Price } \\
\text { (AUD/KW) }\end{array}$ & $\begin{array}{c}\text { Electricity usage } \\
\text { on weekdays (Kw) }\end{array}$ & $\begin{array}{c}\text { Electricity usage } \\
\text { on weekends (Kw) }\end{array}$ & $\begin{array}{c}\text { Early withdrawal } \\
\text { penalty }\end{array}$ & Sale off \\
\hline S1 & 1.40 & 270 & 360 & No & No bonus \\
\hline S2 & 0.70 & 200 & 290 & Yes & Gift \\
\hline S3 & 0.71 & 240 & 400 & No & Free sign up fee \\
\hline S4 & 0.80 & 245 & 320 & No & Free sign up fee \\
\hline S5 & 0.89 & 229 & 350 & No & Gift \\
\hline S6 & 0.98 & 248 & 420 & No & Free sign $4 p$ fee \\
\hline
\end{tabular}

Table 1: Some electricity suppliers

Broker setting: All supplier agents agree that if their electricity is bought by $B_{1}$ through $B R_{1}, B R_{1}$ will receive a reward value called $r$ from supplier agents. In this experiment, the reward is calculated as

$$
r=\text { price } \times 10 \%
$$

It can be seen that if there are more than one supplier agent, which satisfy $B_{1}$ 's requirements, $B R_{1}$ will choose a supplier with the largest reward to $B R_{1}$.

Buyer setting: The buyer agent $B_{1}$ 's requirements are presented as follows. $B_{1}$ 's concession threshold $\lambda$ is set to a value $(50 \%)$ and four considered attributes in $B_{1}$ 's $E E R$ are price, electricity usage on weekdays, electricity usage on weekends and early withdrawal penalty. $B_{1}$ 's acceptability threshold is as $95 \%$ and based on $B_{1}$ 's acceptability threshold, each constraint value of attributes is displayed in 2-5, respectively. In addition, the priority degrees of price, electricity usage on weekdays, electricity usage on weekends and early withdrawal penalty are set to $3,2,1$, and 4 , respectively. Thus, $B_{1}$ 's $E E R$ is shortly presented as follows.

$$
\text { EER }=\left(\begin{array}{cccc} 
& \text { Electricity usage } & \text { Electricity usage } & \text { Early withdrawal } \\
\text { Price } & \text { on weekdays } & \text { on weekends } & \text { penalty } \\
---- & ----- & ----- & ----- \\
\text { under } 0.7 & \text { under } 200 & \text { under } 300 & \text { no } \\
3 & 2 & 1 & 4
\end{array}\right)
$$

\subsection{Experiment results}

In this subsection, we illustrate the experimental results on the trading process between a buyer agent and supplier agents through a broker agent for supplier selection in the power market. 


\begin{tabular}{|c|c|}
\hline & \\
\hline Price (AUD/KW) & Satisfaction degree \\
\hline under 0.7 & $100 \%$ \\
\hline $0.7-1.0$ & $90 \%$ \\
\hline $1.0-1,3$ & $80 \%$ \\
\hline $1.3-1.6$ & $70 \%$ \\
\hline $1.6-1.9$ & $60 \%$ \\
\hline $1.9-2.2$ & $50 \%$ \\
\hline above 2.2 & $40 \%$ \\
\hline \multicolumn{2}{|c|}{ Table 2: Satisfaction degree of price } \\
\hline
\end{tabular}

Table 2: Satisfaction degree of price

\begin{tabular}{|c|c|}
\hline $\begin{array}{c}\text { Electricity usage } \\
\text { on weekends (KW) }\end{array}$ & $\begin{array}{c}\text { Satisfaction } \\
\text { degree }\end{array}$ \\
\hline under 300 & $100 \%$ \\
\hline $300-400$ & $70 \%$ \\
\hline above 400 & $30 \%$ \\
\hline
\end{tabular}

Table 4: Satisfaction degree of electricity usage on weekends

\begin{tabular}{|c|c|}
\hline $\begin{array}{c}\text { Electricity usage } \\
\text { on weekdays (KW) }\end{array}$ & Satisfaction degree \\
\hline under 200 & $100 \%$ \\
\hline $200-220$ & $90 \%$ \\
\hline $220-240$ & $85 \%$ \\
\hline $240-260$ & $80 \%$ \\
\hline $260-280$ & $70 \%$ \\
\hline $280-300$ & $60 \%$ \\
\hline above 300 & $50 \%$ \\
\hline \multicolumn{2}{|c|}{ Table 3: Satisfaction degree of electricity } \\
\hline
\end{tabular}

usage on weekdays

\begin{tabular}{|c|c|}
\hline $\begin{array}{c}\text { Early withdrawal } \\
\text { penalty }\end{array}$ & Satisfaction degree \\
\hline No & $100 \%$ \\
\hline YES & $0 \%$ \\
\hline
\end{tabular}

Table 5: Satisfaction degree of early withdrawal penalty

In the experiment, $B_{1}$ 's acceptability threshold is set at a high value (95\%). This means that it is difficult for the trading process between $B_{1}$ and $\mathbf{S}$ through $B R_{1}$ to achieve an agreement without any relaxation of requirements. Thus, our approach is useful for overcoming this difficulty. In particular, $B_{1}$ uses a relaxation when $B R_{1}$ cannot find any supplier agent, which satisfies $B_{1}$ 's requirements. Supplier agents offer a bonus program to attract $B_{1}$ to purchase their electricity and $B R_{1}$ selects the most suitable supplier for $B_{1}$.

The experimental result is illustrated in Fig. 2. From Fig. 2, we can see that the agreement was achieved through using 8 rounds. The relaxation was applied in rounds 2,4 , and 5 because $B R_{1}$ could not find any supplier agent, which could satisfy $B_{1}$ 's requirements. After the relaxation was used in round $5, B R_{1}$ found that $S_{5}$ could meet $B_{1}$ 's requirements and required $B_{1}$ to verify whether $S_{5}$ was acceptable. Although $S_{5}$ satisfied all constraints of $B_{1}$, the agreement was not achieved because $B_{1}$ 's acceptability degree was $92.5 \%$ for $S_{5}$ which was less than $B_{1}$ 's required acceptability threshold of $95 \%$ in round 6 . So, $B_{1}$ required $B R_{1}$ to find other supplier agent. Then, $B R_{1}$ found $S_{5}$ again with offered bonus and required $B_{1}$ to verify whether $S_{5}$ could be acceptable in round 7. $B_{1}$ calculated the acceptability degree for $S_{5}$ with the offered bonus. The acceptability degree of $S_{5}$ was acceptable and the agreement was achieved in round 8 .

The explanation of such results is (1) The buyer agent used relaxation three times to achieve an agreement with the acceptability threshold $\alpha=95 \%$. If the relaxation was not carried out, the trading process was terminated in round 2 . (2) Supplier agents used a bonus policy to attract the buyer agent to purchase their electricity in round 7 . Thus, the experimental result convinces us that the buyer's relaxation strategy, suppliers' bonus policy and the broker's supplier selection are able to successfully achieve a trading process. 


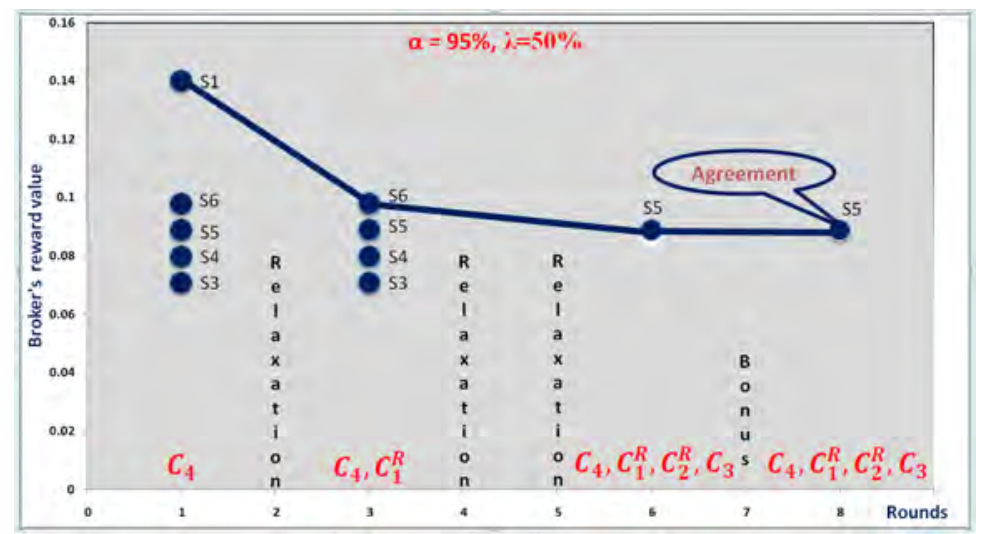

Fig. 2. The experimental result

\subsection{Discussions}

There has been a lot of previous work on regarding the indirect interaction between buyer agents and seller agents through broker agents in e-markets. Sarma et al [11] analyzed market behavior in large networks where buyer agents do not know seller agents and vice-versa. All trading processes between seller agents and buyer agents depend on broker agents. Although they proposed polynomial time algorithms to compute equilibria in networks, their proposed algorithms are only suitable for simple situations in e-markets. The difference between Sarma's work and our work is that all trading processes in our approach are related to three parties involving buyer agents, supplier agents and broker agents. Supplier agents offer a bonus policy to attract buyer agents to purchase their electricity supply. In our approach, broker agents will achieve supplier agents' reward if broker agents sell supplier agents' electricity supply to buyer agents, while Sarma's work does not pay attention to a bonus policy from seller agents to buyer agents and broker agents. Blume et al. [2] modeled the trading phenomenon related to buyer agents, seller agents and trader agents. In their model, trader agents set price strategies, and then buyer agents and seller agents react to the proposed price. Although their model proposed price strategies, it did not incorporate in many features of real markets. The novelty of our approach is that broker agents act as middlemen to find a potential supplier agent to meet the buyer agents' requirements, while Blume's work does not pay attention to how to match buyer agents and seller agents through broker agents.

\section{Conclusion and Future work}

This paper proposed a relaxation strategy with fuzzy constraints to select a potential supplier agent for a buyer agent in the power market through a broker agent. The strategy contains three main components: supplier selection, a fuzzy constraint relaxation and decision making. The proposed approach is novel 
because (1) the trading process between the buyer agent and supplier agents through the broker agent is successfully solved based on fuzzy constraints for multiple attributes of the buyer agent's requirements and power supplier agents; and (2) the buyer agent's relaxation is applied to select a potential supplier agent through the broker agent when the buyer agent's requirements cannot be met by any supplier agents. Also, the experimental results demonstrate the good performance for supplier selection in the power market.

Further work is needed to test the proposed strategy in a real world application and to develop comprehensive strategies with fuzzy constraints to consider the relationships of the buyer agents and the broker agents.

\section{References}

1. M. Bichler. The future of e-markets: multidimensional market mechanisms. Cambridge University Press, 2001.

2. L.E. Blume, D. Easley, J. Kleinberg, and E. Tardos. Trading networks with pricesetting agents. Games and Economic Behavior, 67(1):36-50, 2009.

3. S. Fatima, M. Wooldridge, and N. R. Jennings. An analysis of feasible solutions for multi-issue negotiation involving nonlinear utility functions. In Proceedings of The 8th International Conference on Autonomous Agents and Multiagent SystemsVolume 2, pages 1041-1048. International Foundation for Autonomous Agents and Multiagent Systems, 2009.

4. D.M. Gale and S. Kariv. Financial networks. The American economic review, 97(2):99-103, 2007.

5. W. Ketter, J. Collins, P. Reddy, C. Flath, and M. Weerdt. The 2012 power trading agent competition. ERIM Report Series Reference No. ERS-2012-010-LIS, 2012.

6. X. Luo, N.R. Jennings, N. Shadbolt, H. Leung, and J.H. Lee. A fuzzy constraint based model for bilateral, multi-issue negotiations in semi-competitive environments. Artificial Intelligence, 148(1):53-102, 2003.

7. J. Nicolaisen, V. Petrov, and L. Tesfatsion. Market power and efficiency in a computational electricity market with discriminatory double-auction pricing. Evolutionary Computation, IEEE Transactions on, 5(5):504-523, 2001.

8. F. Ren and M. Zhang. Desire-based negotiation in electronic marketplaces. In Innovations in Agent-Based Complex Automated Negotiations, pages 27-47. Springer, 2011.

9. F. Ren, M. Zhang, and J. Fulcher. Bilateral single-issue negotiation model considering nonlinear utility and time constraint. In New Trends in Agent-Based Complex Automated Negotiations, pages 21-37. Springer, 2012.

10. A. Rubinstein and A. Wolinsky. Middlemen. The Quarterly Journal of Economics, 102(3):581-593, 1987.

11. A.D. Sarma, D. Chakrabarty, and S. Gollapudi. Public advertisement broker markets. In Internet and Network Economics, pages 558-563. Springer, 2007.

12. T.K. Wijaya, K. Larson, and K. Aberer. Matching demand with supply in the smart grid using agent-based multiunit auction. In Communication Systems and Networks (COMSNETS), 2013 Fifth International Conference on, pages 1-6. IEEE, 2013. 